\title{
Generalized statistical mechanics and fully developed turbulence
}

\author{
Christian Beck
}

School of Mathematical Sciences, Queen Mary, University of London, Mile End Road, London E1 4NS.

\begin{abstract}
The statistical properties of fully developed hydrodynamic turbulence can be successfully described using methods from nonextensive statistical mechanics. The predicted probability densities and scaling exponents precisely coincide with what is measured in various turbulence experiments. As a dynamical basis for nonextensive behaviour we consider nonlinear Langevin equations with fluctuating friction forces, where Tsallis statistics can be proved rigorously.
\end{abstract}




\section{Introduction}

The formalism of nonextensive statistical mechanics was introduced by C. Tsallis in 1988 [1] and has been further developed by many others [2]- 6]. Recently, it has been pointed out in a number of papers that the nonextensive approach is in particular useful for the description of stochastic properties of fully developed turbulent flows [7]-15. In this paper we outline the main idea underlying this new statistical mechanics approach to turbulence und compare with various experimental measurements.

First we will summarize the basic idea of the nonextensive formalism and point out some interesting connection to systems with fluctuating temperature or fluctuating energy dissipation (i.e. nonequilibrium systems with a stationary state, see e.g. [16]). As an example where everything can be calculated analytically, we will consider suitable generalizations of Langevin equations with fluctuating friction forces in section 3 [17]. These underly the generalized statistical mechanics in an analogous way as ordinary Langevin equations underly ordinary statistical mechanics. The concrete application to turbulent flows will be described in section 4. Finally, in section 5 we compare with various experimental data in turbulent flows. The agreement turns out to be very good, thus indicating that methods from generalized statistical mechanics are a useful tool for modeling turbulence. Fully developed turbulent flows appear to effectively extremize the Tsallis entropies.

\section{Tsallis statistics and fluctuations}

In the nonextensive statistical mechanics approach, the usual Shannon entropy $S_{1}=-\sum_{i} p_{i} \ln p_{i}$ is replaced by the more general Tsallis entropies

$$
S_{q}=\frac{1}{q-1}\left(1-\sum_{i} p_{i}^{q}\right) .
$$

The $S_{q}$ are related but different from the Renyi entropies (see, e.g., [18]). The $p_{i}$ are probabilities associated with the microstates $i$ of the physical system, and $q \neq 1$ is a parameter, the 'entropic index'. The ordinary Shannon entropy $S_{1}$ is recovered for $q \rightarrow 1$.

Extremizing $S_{q}$ under suitable constraints (see e.g. [3] for a review), just in an analogous way as it is done in ordinary statistical mechanics, one formally obtains a $q$-generalized version of the canonical distributions. The probabilities $p_{i}$ come out of the extremization procedure as

$$
p_{i}=\frac{1}{Z_{q}}\left(1-(1-q) \beta E_{i}\right)^{\frac{1}{1-q}}
$$


where

$$
Z_{q}=\sum_{i}\left(1-(1-q) \beta E_{i}\right)^{\frac{1}{1-q}}
$$

is the partition function, $\beta=1 /(k T)$ is a suitable inverse temperature variable, and the $E_{i}$ denote effective energy levels of the microstates $i$. In the limit $q \rightarrow 1$, ordinary statistical mechanics is recovered, and eq. (2) reduces to the ordinary Boltzmann factor $p_{i} \sim e^{-\beta E_{i}}$. Most formulas of thermodynamics appear to have a simple generalization valid for arbitrary $q$ [3].

Although this is certainly a beautiful mathematical framework, a natural question is whether this type of statistical mechanics is physically realized. Tsallis' original suggestion was that it may have physical applications for equilibrium systems with long-range interactions (see [19] for a recent update). Very recently, a slightly different application was suggested. It was pointed out that the formalism is of particular physical relevance for nonequilibrium systems with fluctuating temperature or fluctuating energy dissipation [10, 17, 20, 21]. The basic idea how fluctuations can generate nonextensive statistics is easily understood: Consider an arbitrary Hamiltonian with energy levels $E_{i}$ of the microstates. Using the integral representation of the gamma function one can easily prove the following formula

$$
\left(1-(1-q) \beta_{0} E_{i}\right)^{\frac{1}{1-q}}=\int_{0}^{\infty} e^{-\beta E_{i}} f(\beta) d \beta,
$$

where

$$
q-1=\frac{2}{n}
$$

and

$$
f(\beta)=\frac{1}{\Gamma\left(\frac{n}{2}\right)}\left\{\frac{n}{2 \beta_{0}}\right\}^{\frac{n}{2}} \beta^{\frac{n}{2}-1} \exp \left\{-\frac{n \beta}{2 \beta_{0}}\right\}
$$

is the probability density of the $\chi^{2}$ (or gamma) distribution of order $n$. It arises in a natural way if $n$ independent Gaussian random variables with average 0 are squared and summed up.

Formula (4) is valid for arbitrary $E_{i}$ and thus universal. The left-hand side of eq. (田) is just the generalized Boltzmann factor emerging out of nonextensive statistical mechanics. The right-hand side is a weighted average over Boltzmann factors of ordinary statistical mechanics. If we consider a nonequilibrim system with fluctuating temperature, then nonextensive behaviour is a consequence of integrating over all possible fluctuating inverse temperatures $\beta$, provided $\beta$ is $\chi^{2}$ distributed.

The constant $\beta_{0}$ in eq. (四) is the average of the fluctuating $\beta$,

$$
\langle\beta\rangle:=\int_{0}^{\infty} \beta f(\beta) d \beta=\beta_{0}
$$


and for the relative variance of the fluctuations one obtains

$$
\frac{\left\langle\beta^{2}\right\rangle-\langle\beta\rangle^{2}}{\langle\beta\rangle^{2}}=\frac{2}{n}
$$

\section{Langevin equation with fluctuating friction forces}

We will now construct a concrete dynamics where Tsallis statistics can be rigorously proved.

Consider a nonlinear Langevin equation of the form

$$
\dot{u}=-\gamma F(u)+\sigma L(t)
$$

where $F(u)=-\frac{\partial}{\partial u} V(u)$ is a nonlinear forcing and $L(t)$ is Gaussian white noise. For the turbulence application, we are mainly interested in power-law potentials of the form $V(u)=C|u|^{2 \alpha}$. Given some fixed ratio $\beta:=\gamma / \sigma^{2}$ of the parameters in the stochastic differential equation (9), this generates a process with the stationary probability density function

$$
p(u \mid \beta)=\frac{\alpha}{\Gamma\left(\frac{1}{2 \alpha}\right)}(C \beta)^{\frac{1}{2 \alpha}} \exp \left\{-\beta C|u|^{2 \alpha}\right\} .
$$

We now allow the parameters $\gamma$ and $\sigma$ in eq. (9) to fluctuate as well. Let us assume that either $\gamma$ or $\sigma$ or both fluctuate in such a way that $\beta=\gamma / \sigma^{2}$ is $\chi^{2}$-distributed with degree $n$. For example, we can generate this $\chi^{2}$-distribution by yet another set of Langevin equations, say

$$
\dot{X}_{j}=-\Gamma X_{j}+L_{j}(t) \quad(j=1, \ldots, n),
$$

and by defining

$$
\beta=\frac{\gamma}{\sigma^{2}}:=\sum_{j=1}^{n} X_{j}^{2} .
$$

On a long time scale this set of linear Langevin equations clearly generates independent Gaussian random variables $X_{j}$ with average 0 (provided $\Gamma=$ const), hence $\beta$ is $\chi^{2}$-distributed with density (6).

Assume that the time scale on which $\beta$ fluctuates is very large, so that for a given $\beta$ local equilibrium described by $p(u \mid \beta)$ is achieved in eq. (9). In the long-

term run we have to take into account all fluctuating values of $\beta$. This means 
we will ultimately observe the marginal distribution $p(u):=\int p(u \mid \beta) f(\beta) d \beta$ being generated by eq. (9). This integral can be performed exactly and one obtains

$$
p(u)=\frac{1}{Z_{q}} \frac{1}{\left(1-(1-q) \tilde{\beta} C|u|^{2 \alpha}\right)^{\frac{1}{q-1}}},
$$

where

$$
Z_{q}^{-1}=\alpha(C(q-1) \tilde{\beta})^{\frac{1}{2 \alpha}} \cdot \frac{\Gamma\left(\frac{1}{q-1}\right)}{\Gamma\left(\frac{1}{2 \alpha}\right) \Gamma\left(\frac{1}{q-1}-\frac{1}{2 \alpha}\right)}
$$

and

$$
\begin{aligned}
q & =1+\frac{2 \alpha}{\alpha n+1} \\
\tilde{\beta} & =\frac{2 \alpha}{1+2 \alpha-q} \beta_{0} .
\end{aligned}
$$

\section{Application to turbulent flows}

Let us now come to our main physical example, fully developed turbulence. Let $u$ in eq. (9) represent a local velocity difference in a fully developed turbulent flow as measured on a certain scale $r$. It is well known that the dissipation fluctuates in turbulent flows, so our model makes sense. The parameter $\beta=\gamma / \sigma^{2}$ of the stochastic differential equation is an a priori unknown function of the energy dissipation in the flow. Let us consider a model where

$$
\beta=\frac{\gamma}{\sigma^{2}}=\epsilon_{r} \tau \Lambda
$$

Here $\epsilon_{r}$ is the (fluctuating) energy dissipation rate averaged over $r^{3}$ and $\tau$ is a typical time scale during which energy is transferred. $\Lambda$ is a constant with dimension length ${ }^{4} /$ time $^{4}$, its value is irrelevant for the following. Both $\epsilon_{r}$ and $\tau$ can fluctuate, and we assume that $\beta \sim \epsilon_{r} \tau$ is $\chi^{2}$-distributed, thus ending up with the dynamical model of the previous section.

We remind those readers not familiar with turbulence that the averaged energy dissipation rate $\epsilon_{r}(\vec{x}, t)$ in a volume $V$ of size $r^{3}$ is defined as

$$
\epsilon_{r}(\vec{x}, t)=\frac{1}{r^{3}} \int_{V} \epsilon(\vec{x}+\vec{r}, t) d^{3} r
$$

where

$$
\epsilon(\vec{x}, t)=\frac{1}{2} \nu \sum_{i, j}\left(\frac{\partial v_{i}}{\partial x_{j}}+\frac{\partial v_{j}}{\partial x_{i}}\right)^{2}
$$


is the instantaneous dissipation, $\nu$ is the kinematic viscosity, and $v_{i}$ is the velocity fluctuation (the deviation from the mean velocity) in $i$ direction [22]. The physical idea of considering the generalized Langevin equation (9) with $\beta \sim \epsilon_{r} \tau$ is that a test particle in the turbulent flow moves for a while in a certain region with a given $\epsilon_{r}$, then moves to another region with another $\epsilon_{r}$, and so on. In this picture, we actually associate the fluctuations of $\beta$ with spatial fluctuations in a spatially extended system.

At the smallest scale of the turbulent flow, the Kolmogorov length scale $\eta=$ $\left(\nu^{3} / \epsilon\right)^{1 / 4}$, we can give a theoretical prediction of the entropic index $q$. Here we can write

$$
\beta=\epsilon \tau_{\eta} \Lambda=(\epsilon \nu)^{1 / 2} \Lambda=\left(u_{\eta}\right)^{2} \Lambda,
$$

where $\tau_{\eta}:=(\nu / \epsilon)^{1 / 2}$ denotes the Kolmogorov time and $u_{\eta}:=\eta / \tau_{\eta}=(\nu \epsilon)^{1 / 4}$ is the Kolmogorov velocity (see, e.g., [22]). If $\epsilon$ fluctuates then all these quantities fluctuate as well. Due to the three spatial directions one can actually define 3 independently fluctuating Kolmogorov velocities $u_{\eta}^{j}, j=1,2,3$ such that $u_{\eta}=$ $\left|\vec{u}_{\eta}\right|=\sqrt{\sum_{i=1}^{3}\left(u_{\eta}^{j}\right)^{2}}$. The 3 components $u_{\eta}^{j}$ describe the flow of energy into the three different space directions. The simplest model assumption is that these Kolmogorov velocities are Gaussian with average 0 . This means, we identify $X_{j}=$ $\sqrt{\Lambda} u_{\eta}^{j}$ in eq. (12). Hence at the smallest scale the 3 space dimensions lead to $n=3$ or, using eq. (15), $q=\frac{3}{2}$ if $\alpha=1$.

Note that our approach allows us to view the fluctuations of $\epsilon$ at the Kolmogorov scale in terms of a (hypothetical) ordinary Brownian particle of mass $M$ that is subjected to ordinary thermal noise of temperature $T$. The fluctuating velocity $\vec{V}$ of this constructed Brownian particle coincides with the fluctuating vector $\vec{u}_{\eta}$ of Kolmogorov velocities. The Brownian particle just absorbs the turbulent energy flow at the Kolmogorov scale. It bridges the gap between thermal and macroscopic description. Equipartition of energy yields

$$
\frac{1}{2} M\left\langle\left(u_{\eta}^{1}\right)^{2}\right\rangle=\frac{1}{2} k T \text {. }
$$

Using eq. (20) we can estimate the mass of our energy-absorbing Brownian particle as

$$
M=\frac{3 k T}{\nu^{1 / 2}\left\langle\epsilon^{1 / 2}\right\rangle}
$$

\section{Comparison with experiments}

The quality of agreement of the generalized canonical distributions (13) with experimentally measured densities is remarkable. To start with, Fig. 1 shows probability 
densities of longitudinal velocity differences as measured by Swinney et al. in a turbulent Taylor-Couette flow on various scales $r[8]$. They are very well fitted by eq. (13) choosing an $r$-dependent $q$ and $\alpha=2-q$. In [8], in total 152 different densities were evaluated, varying the spatial scale $r$ and the Reynolds numbers $R e$. Previous fitting attempts using e.g. stretched exponentials do not yield anything of comparable precision.

Next, Fig. 2 shows the results of measurements in another turbulence experiment. Here we see a histogram of the acceleration $a$ of a test particle advected by the turbulent flow (measurements of Lagrangian turbulence by Bodenschatz et al. [23]). The acceleration has been rescaled to variance 1 . It can be regarded as a velocity difference on the smallest time scale that makes sense in turbulence, the Kolmogorov time scale. Hence our consideration in the previous section predicts Tsallis statistics with $q=3 / 2$ provided $\alpha=1$. Formula (13) yields a very good fit of the measured distributions (solid line in Fig. 2). Note that we do not use any fitting parameter in this plot, since $q=3 / 2$ is predicted, and variance 1 implies $\tilde{\beta} C=4$. On the contrary, in their paper [23] Bodenschatz et al. fit their data with the density function

$$
p(x)=c_{1} \cdot \exp \left\{-\frac{x^{2}}{c_{2}+c_{3}|x|^{c_{4}}}\right\},
$$

i.e. a somewhat arbitrary modification of a stretched exponential with several parameters $c_{i}$. It yields a fit of similar quality as our formula but requires much more free parameters. Moreover, there is no theoretical reason whatsoever for a density of the form (23), whereas nonextensive statistical mechanics, leading to eq. (13), is a well-defined theoretical concept distinguished by an extremization principle and generalized Khinchin axioms [2].

Also scaling exponents can be evaluated using nonextensive methods. The scaling exponents $\zeta_{m}$ of moments of radial velocity differences $u=v(x+r)-v(x)$ measured at distance $r$ in fully developed turbulent flows have a long history (see, e.g., 24, 25]). In the inertial range one observes scaling behaviour of the form

$$
\left\langle|u|^{m}\right\rangle \sim r^{\zeta_{m}}
$$

Recently it has been suggested to calculate the $\zeta_{m}$ using nonextensive methods [7, 9, 12, 14. One approach is based on an extension of the multifractal model of turbulence [12] 14], another one on a combination of the approach described here with so-called extended self-similarity (ESS) [26]. A typical result of the latter approach is shown in Fig. 3 (see 9] for more details). The nonextensive model clearly reproduces the experimental data of scaling exponents very well. This is, by the way, not even surprising, because if the densities (13) perfectly coincide with the experimental densities than the moments must coincide as well. The moments 
of the generalized canonical distribution (13) can be analytically evaluated, they are given by

$$
\left\langle|u|^{m}\right\rangle=\int_{-\infty}^{+\infty}|u|^{m} p(u) d u=\left\{\frac{1}{(q-1) \tilde{\beta} C}\right\}^{\frac{m}{2 \alpha}} \frac{B\left(\frac{m+1}{2 \alpha}, \frac{1}{q-1}-\frac{m+1}{2 \alpha}\right)}{B\left(\frac{1}{2 \alpha}, \frac{1}{q-1}-\frac{1}{2 \alpha}\right)},
$$

where $B(x, y)$ denotes the beta function.

Finally, one may also try to understand the scale-dependence of the entropic index $q(r)$, which has been measured very precisely in [8]. Nonextensive statistical mechanics does not say anything on the function $q(r)$. For the $r$-dependence one needs additional theoretical input. One possibility was recently suggested in [21]. The basic idea is as follows. The Tsallis entropies are non-extensive, i.e. for independent subsystems I and II composed to a single system I+II one has [1, 3, 5]

$$
S_{q}^{I}+S_{q}^{I I}+(1-q) S_{q}^{I} S_{q}^{I I}=S_{q}^{I+I I} .
$$

The observation, however, is that it is possible to make the Tsallis entropies quasiadditive by choosing different entropic indices at different scales. I.e., given a certain $q$ for two small independent subsystems I and II we may choose another $q^{\prime}$ for the larger, composed system I+II such that

$$
S_{q}^{I}+S_{q}^{I I}=S_{q^{\prime}}^{I+I I} .
$$

This property is called quasi-additivity [21]. In turbulence, $q$ is close to 1 , so that a perturbative expansion in $q-1$ makes sense. The final result of a calculation in 21] is that if higher-order contributions in $q-1$ are neglected then quasi-additivity implies a power law for $1 /(q-1)$ as a function of $r$,

$$
\frac{1}{q-1} \sim\left(\frac{r}{\eta}\right)^{\delta}
$$

provided $r$ is large enough. The exponent $\delta$ is non-universal, at least for finite Reynolds numbers. Fig. 4 shows that the Swinney data indeed confirm a power law.

To summarize, the nonextensive approach yields a very useful new method to describe the most important statistical properties of fully developed turbulence. It is in very good agreement with experimental measurements.

\section{References}

[1] C. Tsallis, J. Stat. Phys. 52, 479 (1988) 
[2] S. Abe, Phys. Lett. 271A, 74 (2000)

[3] C. Tsallis, Braz. J. Phys. 29, 1 (1999)

[4] S. Abe, S. Martínez, F. Pennini, and A. Plastino, Phys. Lett. 281A, 126 (2001)

[5] S. Abe, Phys. Rev. 63E, 061105 (2001)

[6] C. Tsallis, R.S. Mendes and A.R. Plastino, Physica 261A, 534 (1998)

[7] C. Beck, Physica 277A, 115 (2000)

[8] C. Beck, G.S. Lewis and H.L. Swinney, Phys. Rev. 63E, 035303(R) (2001)

[9] C. Beck, Physica 295A, 195 (2001)

[10] C. Beck, Phys. Lett. 287A, 240 (2001)

[11] B.K. Shivamoggi, C. Beck, J. Phys. 34A, 4003 (2001)

[12] T. Arimitsu and N. Arimitsu, J. Phys. 33A, L235 (2000)

[13] T. Arimitsu and N. Arimitsu, Phys. Rev. 61E, 3237 (2000)

[14] T. Arimitsu and N. Arimitsu, Prog. Theor. Phys. 105, 355 (2001)

[15] F.M. Ramos, R.R. Rosa and C. Rodrigues Neto, Physica 295A, 250 (2001)

[16] E.G.D. Cohen, Physica 240A, 45 (1997)

[17] C. Beck, Dynamical foundations of nonextensive statistical mechanics, to appear in Phys. Rev. Lett. (2001)

[18] C. Beck and F. Schlögl, Thermodynamics of Chaotic Systems, Camdridge University Press, Cambridge (1993)

[19] V. Latora, A. Rapisarda and C. Tsallis, cond-mat/0103540

[20] G. Wilk and Z. Wlodarczyk, Phys. Rev. Lett. 84, 2770 (2000)

[21] C. Beck, Non-additivity of Tsallis entropies and fluctuations of temperature, cond-mat/0105371

[22] S.B. Pope, Turbulent Flows, Cambridge University Press, Cambridge (2000)

[23] A. La Porta, G.A. Voth, A.M. Crawford, J. Alexander, and E. Bodenschatz, Nature 409, 1017 (2001)

[24] T. Bohr, M.H. Jensen, G. Paladin and A. Vulpiani, Dynamical Systems Approach to Turbulence, Cambridge University Press, Cambridge (1998)

[25] U. Frisch, Turbulence: the Legacy of A.N. Kolmogorov, Cambridge University Press, Cambridge (1995) 
[26] R. Benzi, S. Ciliberto, R. Tripiccione, C. Baudet, F. Massaioli and S. Succi, Phys. Rev. 48E, R29 (1993)

[27] G.S. Lewis and H.L. Swinney, Phys. Rev. 59E, 5457 (1999)

[28] F. Anselmet, Y. Gagne, E.J. Hopfinger and R. Antonia, J. Fluid Mech. 140, $63(1984)$

[29] G. Stolovitzky, K. R. Sreenivasan and A. Juneja, Phys. Rev. 48E, R3217 (1993)

[30] F. Belin, P. Tabeling and H. Willaime, Physica 93D, 52 (1996)

\section{Figure captions}

Fig. 1 Experimentally measured probability densities in a Taylor-Couette flow as measured by Swinney et al. [8] for $r=23,46,93,208,399,830 \eta$ and comparison with formula (13) with $q=1.150,1.124,1.106,1.086,1.066,1.055(\alpha=2-q)$. The $j$-th density is shifted by $-j$ units. All densities are rescaled to variance 1 .

Fig. 2 Experimentally measured measured probability density of the acceleration $x:=a /\left\langle a^{2}\right\rangle^{1 / 2}$ in Lagrangian turbulence as measured by Bodenschatz et al. 23] for Reynolds numbers $R_{\lambda}=200,690,970$ and comparison with formula (13) with $q=3 / 2$ and $\alpha=1$.

Fig. 3 Scaling exponents $\zeta_{m}$ as measured in five different experiments [26]-30] and comparison with the nonextensive prediction of [9].

Fig. $4(q-1)^{-1}$ as a function of $r$. The data points are the $q$-values obtained from the the measured densities of Swinney et al. [8], the straight line corresponds to a power law as predicted by quasi-additivity. The observed exponent is $\delta=0.30$.

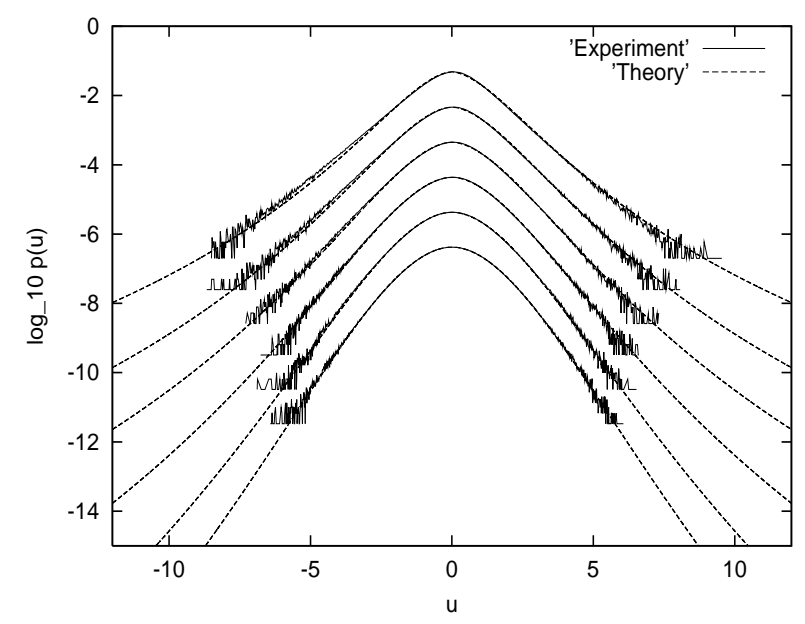



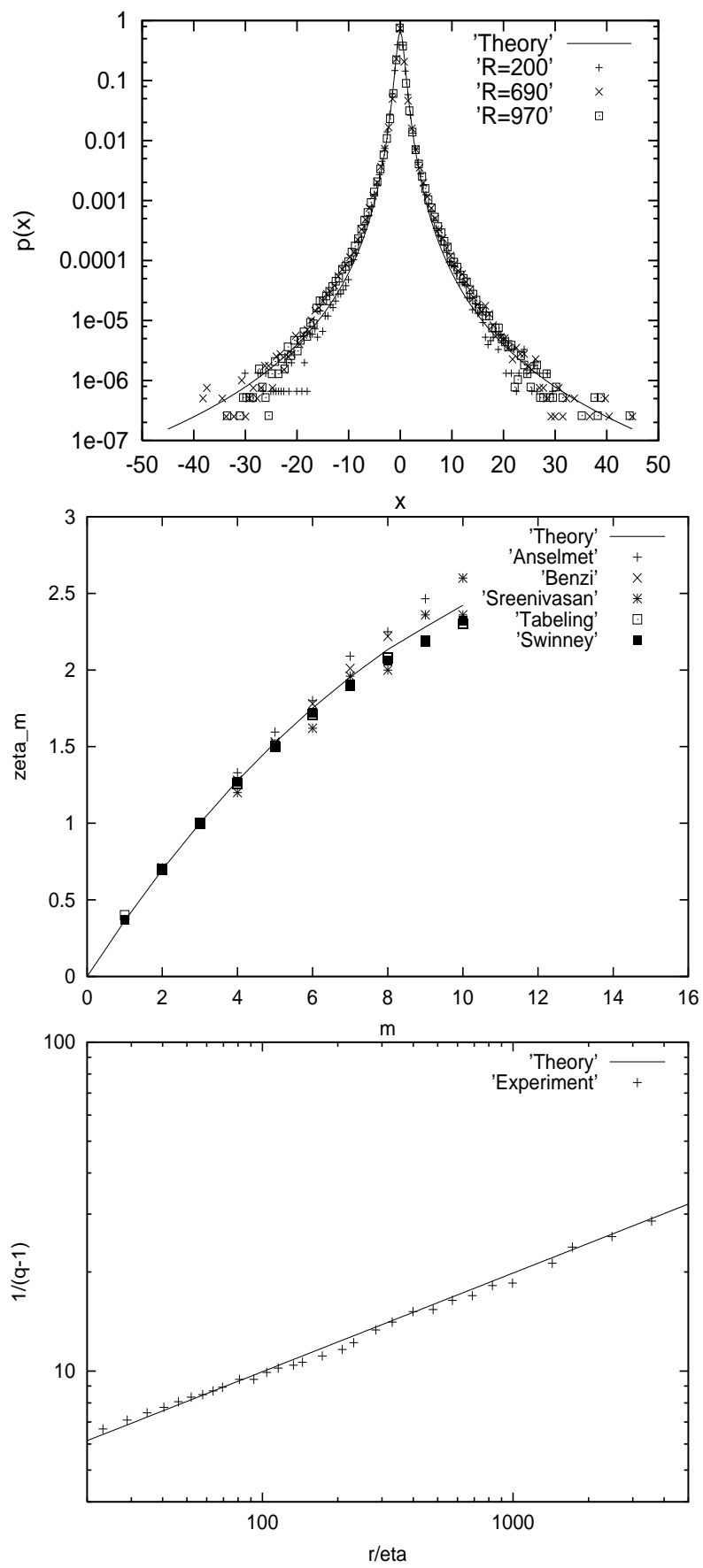